\title{
Effect of softening of cement asphalt mortar on vehicle operation safety and track dynamics"
}

\author{
Jian HAN $^{1}$, Guo-tang ZHAO ${ }^{1,2}$, Xin-biao XIAO ${ }^{1}$, Ze-feng WEN ${ }^{1}$, Qing-hua GUAN ${ }^{1}$, Xue-song JIN ${ }^{\dagger * 1}$ \\ ( ${ }^{1}$ State Key Laboratory of Traction Power, Southwest Jiaotong University, Chengdu 610031, China) \\ $\left({ }^{2}\right.$ China Railway Corporation, Beijing 100844, China) \\ †E-mail: xsjin@home.swjtu.edu.cn
}

Received Apr. 4, 2015; Revision accepted Aug. 31, 2015; Crosschecked Nov. 10, 2015

\begin{abstract}
Cement asphalt mortar (CAM) softening is a common phenomenon that results from ageing and rain soaking when a high-speed railway is in service. CAM softening seriously affects vehicle operation safety and track dynamics. In this paper, a 3D coupling dynamic model of a vehicle and a China railway track system I (CRTS-I) slab track is developed. By using the proposed model, the wheel-rail contact forces, derailment coefficient, wheelset loading reduction ratio, and the track displacements are calculated to study the influences of CAM softening on the dynamic characteristics of a vehicle-track system. A track-subgrade finite difference model is developed to study the effect of CAM softening on track damage. The results show that track interface shear failure develops when the CAM softening coefficients reach 10-100. The CAM softening coefficient should not be less than 1000 , otherwise a high-speed running vehicle may risk derailment.
\end{abstract}

Key words: High-speed railway, Cement asphalt mortar (CAM) softening, Vehicle-track coupling dynamics, Operation safety, Track damage

doi: $10.1631 /$ jzus.A1500080

Document code: A

CLC number: U270

\section{Introduction}

In the operation of high-speed trains, different degrees of damage are suffered by the cement asphalt mortar (CAM) that forms the filling layer between the slab and the concrete base (Lin, 2009; Liu, 2013). The damage includes cracks, shelling, ageing, and rain soaking (Fig. 1). Much research work on vehicletrack coupling dynamics and track-subgrade dynamics was carried out (Chen et al., 2014; Ling et al., 2014; Zhong et al., 2014). However, there have been few studies of vehicle-track coupling systems that consider CAM damage. Xiang et al. (2009) studied the effect of a voided slab induced by the

\footnotetext{
${ }^{\ddagger}$ Corresponding author

* Project supported by the National Natural Science Foundation of China (Nos. U1134202 and 51305360), the National Basic Research Program (973 Program) of China (No. 2011CB711103), and the 2015 Doctoral Innovation Funds of Southwest Jiaotong University, China (D) ORCID: Jian HAN, http://orcid.org/0000-0002-7891-7164 (c) Zhejiang University and Springer-Verlag Berlin Heidelberg 2015
}

deterioration of the CAM layer on vibration responses of a slab track at variable vehicle speeds. Based on the perspective of the system energy, he used the Wilson- $\theta$ numerical integral method to solve the track vibration equations. Wang et al. (2014) analysed the effect of CAM debonding on the dynamic properties of a CRTS-II slab track, using LS-DYNA to solve the dynamic equations. Both Xiang et al. (2009) and Wang et al. (2014) treated the rail as a continuous Euler beam, and their models considered only vertical vibration. Zhu and Cai (2014) investigated interface damage and its effect on vibrations of a slab track under different temperature and vehicle dynamic loads. The loads were obtained using the developed vehicle-track coupling dynamic model and the track model was developed using ABAQUS software. The model assumed that the influence of temperature is important to CAM damage after a period of time. However, when CAM damage had already occurred, its effect on train running safety was not discussed. 
The CAM softening not only leads to track structural failure but also becomes a potential factor responsible for increasing the probability of vehicle derailment. Zhou and Shen (2013) and Xiao et al. (2007) studied the effect of disabled fastening systems or unsupported sleepers on ballast tracks on vehicle derailment using a vehicle-track coupling dynamic model. CAM damage is particularly common in slab tracks. It is important to study the influences of CAM softening on the dynamic characteristics of a $3 \mathrm{D}$ vehicle-track system because CAM damage endangers the safety of train operation, especially for curved lines.

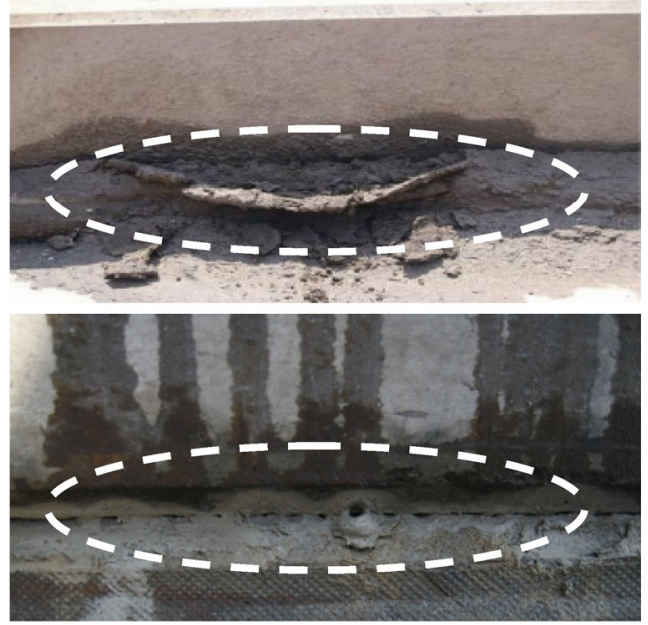

Fig. 1 CAM damage (softening). Reprinted from (Zhu, 2014), Copyright 2014, with permission from Elsevier

CAM softening seriously affects vehicle operation safety and track interface shear failure. In this paper, a 3D coupling dynamic model of a vehicle and a CRTS-I slab track is developed. The vehicle runs on a curved track at $300 \mathrm{~km} / \mathrm{h}$. Using the proposed model, the wheel-rail contact forces, derailment coefficient, wheelset loading reduction ratio, and the track displacements are calculated to study the influences of CAM softening on the dynamic characteristics of the vehicle-track system. A track-subgrade finite difference model is developed to investigate the effect of CAM softening on slab stress and track interface failure.

\section{Coupling dynamic model of vehicle and CRTS-I slab track}

Fig. 2 illustrates the coupling dynamic model of a high-speed vehicle and the CRTS-I slab track to study the effect of CAM softening on the dynamic behavior of a vehicle-track system. In the numerical simulation, different degrees of CAM softening are considered under one slab. A moving rail-support is adopted as a new vehicle-track coupling interface excitation model (called the "Tracking Window") (Jin and Wen, 2008; Xiao et al., 2011; Jin, 2014). This excitation model is closer to a real moving vehicle under the excitation of discrete sleepers, and saves a lot of computation time. The vehicle-track coupling system equations are solved by means of a new explicit integration method (Zhai, 1996).

\subsection{Dynamic model of vehicle subsystem}

The high-speed railway vehicle is considered as a rigid multi-body model, in which the car body is supported by two double-axle bogies with the primary and the secondary suspension systems. For the connecting parts (the primary vertical damper, the secondary lateral damper, the secondary yaw damper, and the lateral stopping block) with nonlinear characteristics, a piecewise linear simulation is used. Each component of the vehicle has six degrees of freedom (DOFs): longitudinal motion, lateral motion, vertical motion, roll angle, yaw angle, and pitch angle (Fig. 2). The vehicle has a total of 42 DOFs. Based on the coordinate system, moving along the track at the constant speed of the vehicle, the equation of the vehicle subsystem can be described in the second-order differential equation in the time domain as follows:

$$
\boldsymbol{M}_{\mathrm{v}} \ddot{\boldsymbol{u}}_{\mathrm{v}}+\boldsymbol{C}_{\mathrm{v}} \dot{\boldsymbol{u}}_{\mathrm{v}}+\boldsymbol{K}_{\mathrm{v}} \boldsymbol{u}_{\mathrm{v}}=\boldsymbol{F}_{\mathrm{v}}
$$

where $\boldsymbol{M}_{\mathrm{v}}$ is the mass matrix of the vehicle, and $\boldsymbol{C}_{\mathrm{v}}$ and $\boldsymbol{K}_{\mathrm{v}}$ are the damping and the stiffness matrices. $\boldsymbol{u}_{\mathrm{v}}, \dot{\boldsymbol{u}}_{\mathrm{v}}$, and $\ddot{\boldsymbol{u}}_{\mathrm{v}}$ are the vectors of displacement, velocity, and acceleration, respectively, of the vehicle subsystem, and $\boldsymbol{F}_{\mathrm{v}}$ is the vector of generalized loads acting on the vehicle subsystem.

\subsection{Dynamic model of slab track subsystem}

The dynamic model of the slab track subsystem includes rails, fastener systems, slabs, CAM layers, and concrete base (Fig. 2). The rail is treated as a continuous Timoshenko beam resting on rail pads, and the lateral, vertical, and torsion motions of rails are simultaneously taken into account (Xiao et al., 2008). The slabs and the concrete base are modeled 


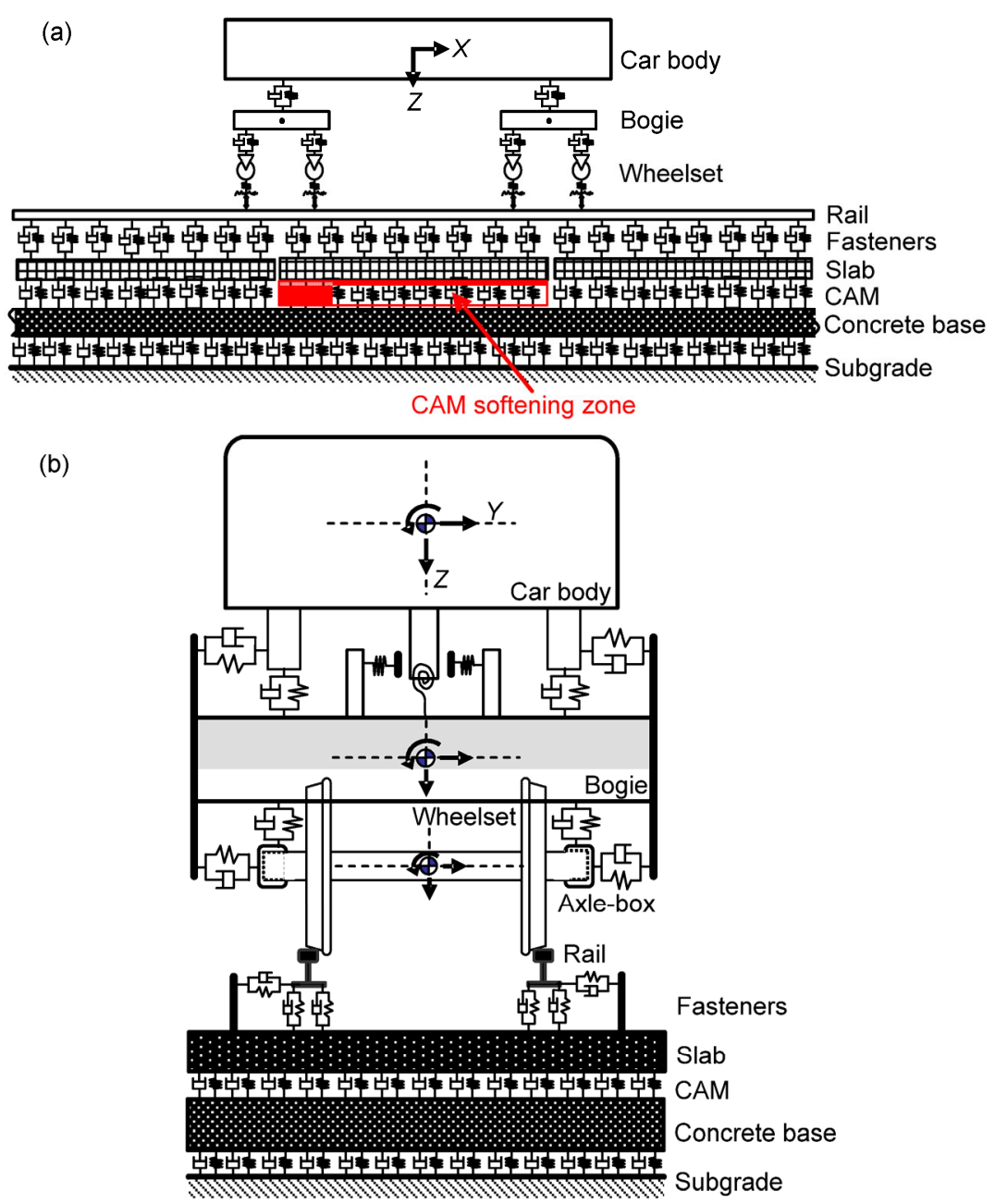

Fig. 2 Vehicle-track coupling dynamic model

(a) Elevation; (b) End view

using the 3D finite element method. The rail fastener systems and the CAM layer are modeled using periodic discrete viscoelastic units. The finite element model of the slab has 20600 solid elements and 26520 DOFs. The length of the slab is $4.962 \mathrm{~m}$. The geometric dimensions of its cross section are $2.4 \mathrm{~m}$ $\times 0.19 \mathrm{~m}$. The vibration of the slab can be easily described in the second-order differential equation in terms of generalized coordinates, as expressed by Eq. (2). Modal analysis of the slab is carried out by means of ANSYS to obtain 20 order modes, by which Eq. (2) is decoupled and solved according to the modal superposition principle, as follows:

$$
\boldsymbol{M}_{\mathrm{s} i} \ddot{\boldsymbol{u}}_{\mathrm{s} i}+\boldsymbol{C}_{\mathrm{si} i} \dot{\boldsymbol{u}}_{\mathrm{s} i}+\boldsymbol{K}_{\mathrm{si}} \boldsymbol{u}_{\mathrm{s} i}=\boldsymbol{F}_{\mathrm{rs} i}+\boldsymbol{F}_{\mathrm{sc} i},
$$

where $\boldsymbol{M}_{\mathrm{s} i}, \boldsymbol{C}_{\mathrm{s} i}$, and $\boldsymbol{K}_{\mathrm{s} i}$ are the mass, damping, and stiffness matrices, respectively, of the $i$ th slab. $\ddot{\boldsymbol{u}}_{\mathrm{si}}, \dot{\boldsymbol{u}}_{\mathrm{s} i}$, and $\boldsymbol{u}_{\mathrm{si}}$ are the acceleration vector, velocity vector, and displacement vector, respectively. $\boldsymbol{F}_{\mathrm{r} s i}$ is the load vector between the rail and the $i$ th slab, and $\boldsymbol{F}_{\text {sc } i}$ is the load vector between the slab and the concrete base.

The model of the concrete base is similar to that of the slab. The concrete base model has 433956 solid elements and 515424 DOFs. The length of the concrete base is $60 \mathrm{~m}$. The geometric dimensions of its cross section are $0.3 \mathrm{~m} \times 3 \mathrm{~m}$.

\subsection{Model of wheel-rail interaction in rolling contact}

Wheel/Rail dynamic interaction modeling is the key to the vehicle-track coupling dynamic model. The calculation of wheel/rail contact forces includes a 
normal model and a tangent model. The normal model, which characterizes the relationship law of a normal load and deformation between the wheel and rail, is described by a nonlinear Hertz contact spring with a unilateral restraint:

$$
N(t)= \begin{cases}{\left[\frac{1}{G_{\text {Hertz }}} Z_{\mathrm{wrnc}}(t)\right]^{3 / 2},} & Z_{\mathrm{wrnc}}(t)>0, \\ 0, & Z_{\mathrm{wrnc}}(t) \leq 0,\end{cases}
$$

where $G_{\text {Hertz }}$ is the wheel/rail contact constant $\left(\mathrm{m} / \mathrm{N}^{2 / 3}\right)$, which can be obtained using the Hertz contact theory. $Z_{\mathrm{wrnc}}(t)$ is the normal amount of compression at the wheel/rail contact point. $Z_{\mathrm{wrnc}}(t)$ is strictly defined as an approach between two distant points, one belonging to the wheel, and the other belonging to the rail. The wheel and the rail are assumed to be an elastic half-space. This approach is confined to the normal direction at the contact point of the wheel and the rail. $Z_{\text {wrnc }}(t)>0$ indicates the wheel/rail in contact, and $Z_{\text {wrnc }}(t) \leq 0$ indicates their separation.

The tangential wheel-rail creep forces are calculated using the Shen-Hedrick-Elkins non-linear theory (Shen et al., 1983). In this paper, when calculating the dynamic response of the vehicle-track, the tracing-curve-method (Chen and Zhai, 2004) is adopted to locate the wheel-rail spatial contact geometry. This can greatly reduce the computational time.

\subsection{CAM softening in the vehicle-track coupling dynamic model}

CAM softening, including CAM ageing or rain soaking, is considered. CAM softening leads to changes in the vertical and lateral supporting stiffnesses of the slab, and becomes a potential factor responsible for increasing the probability of vehicle derailment.

CAM softening is simulated by changing the stiffness coefficient of the CAM layer, i.e., the parameters considered are multiplied by "softening coefficients" in the coupled vehicle-track model. The damping used in this paper is assumed to be structural damping. So the same softening coefficient is applied to damping, as shown in Eqs. (4) and (8).

$$
\left\{\begin{array}{l}
K_{\mathrm{scl}}^{\prime}=K_{\mathrm{scl}} / \lambda_{\mathrm{scl}}, C_{\mathrm{scl}}^{\prime}=C_{\mathrm{scl}} / \lambda_{\mathrm{scl}}, 1<\lambda_{\mathrm{scl}}<+\infty \\
K_{\mathrm{scv}}^{\prime}=K_{\mathrm{scv}} / \lambda_{\mathrm{scv}}, C_{\mathrm{scv}}^{\prime}=C_{\mathrm{scv}} / \lambda_{\mathrm{scv}}, 1<\lambda_{\mathrm{scv}}<+\infty
\end{array}\right.
$$

where $K_{\text {scl }}^{\prime}$ is the softening lateral stiffness, $K_{\text {scl }}$ is the original lateral stiffness, $C_{\text {scl }}^{\prime}$ is the softening lateral damping, $C_{\mathrm{scl}}$ is the original lateral damping, $K_{\mathrm{scv}}^{\prime}$ is the softening vertical stiffness, $K_{\text {scv }}$ is the original vertical stiffness, $C_{\text {scv }}^{\prime}$ is the softening vertical damping, $C_{\mathrm{scv}}$ is the original vertical damping, $\lambda_{\mathrm{scl}}$ is the lateral softening coefficient, and $\lambda_{\text {scv }}$ is the vertical softening coefficient.

\subsection{Evaluation criteria of railway vehicle derailment}

At present, two important criteria are widely used to evaluate the dynamic behavior and safety operation of high-speed trains (Xiao et al., 2007; 2014; Zhou and Shen, 2013). One is Nadal's criterion (derailment coefficient), denoted by Eq. (5), and the other is the wheelset loading reduction, indicated by Eq. (6):

$$
\begin{aligned}
& \left(\frac{L}{V}\right)_{\text {Critical }}=\frac{\tan \delta_{\text {max }}-\mu}{1+\mu \tan \delta_{\text {max }}}, \\
& \frac{\Delta V}{V}=\frac{\frac{1}{2}\left(V_{\mathrm{L}}-V_{\mathrm{R}}\right)}{\frac{1}{2}\left(V_{\mathrm{L}}+V_{\mathrm{R}}\right)}=\frac{V_{\mathrm{L}}-V_{\mathrm{R}}}{V_{\mathrm{L}}+V_{\mathrm{R}}},
\end{aligned}
$$

where $\delta_{\max }$ is the maximum flange angle of the wheel, and $\mu$ indicates the friction coefficient between the wheel and the rail. $L$ and $V$ denote the lateral and vertical forces, respectively, of the wheel and the rail, and $\Delta V$ indicates the normal loading difference between the left and right wheels of the same wheelset.

\section{Track/Subgrade coupling model}

\subsection{Finite difference model of slab track and subgrade}

Fig. 3 shows the 3D finite difference model of the CRTS-I slab track system and its subgrade built in this study. The slab track includes three layers: the slab, the CAM, and the concrete base. The subgrade includes three layers: the upper, middle, and bottom layers. The layers have different properties (Table 1). The constitutive relation of the CAM is the Mohr-Coulomb elastic-plastic model. Those of the other parts are linear elastic models. Table 2 shows the material parameters of the CRTS-I slab track 
Table 1 Material parameters of subgrade components

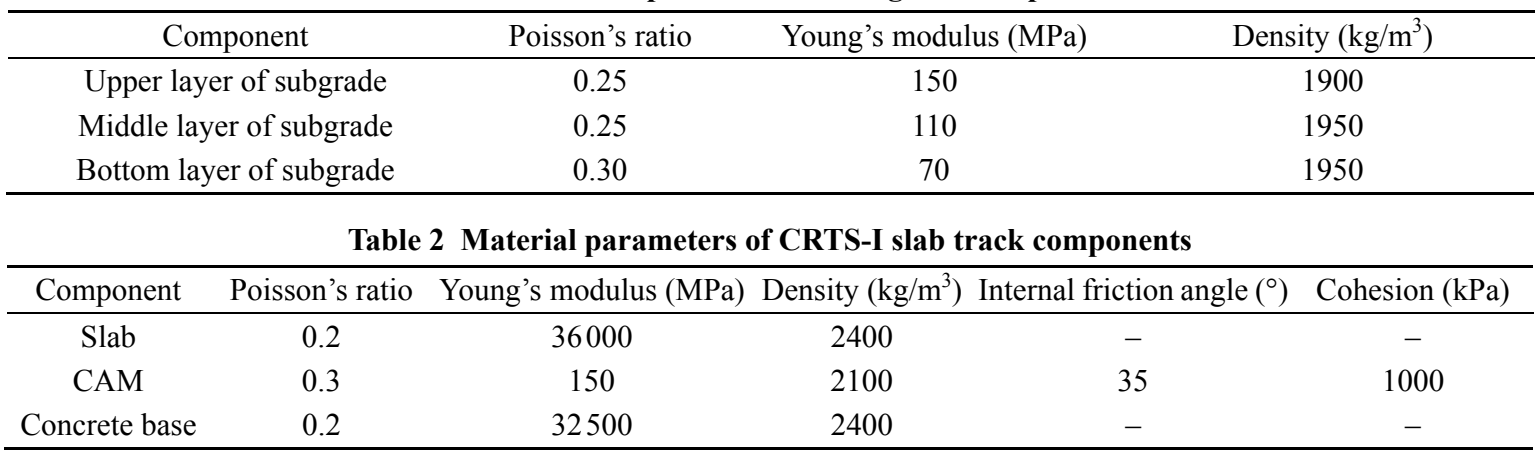

components. A viscoelastic artificial boundary (Liu, et al., 2006) was applied to the bottom and sides along the longitudinal direction of the subgrade. This can characterize the real behaviour of the subgrade bottom support, and avoid wave reflection on the boundary and model infinite track length along the longitudinal direction. The boundary is simulated using a normal and tangential spring and damping. One end of the spring-damping is connected to the subgrade boundary, and the other end is fixed. Eq. (7) describes their stiffness and damping. The subgrade slope is free. There are three slabs in Fig. 3. The middle slab was chosen for the analysis.

$$
\left\{\begin{array}{l}
K_{\mathrm{t}}=0.5 G \Delta s / R, \\
K_{\mathrm{n}}=G \Delta s / R, \\
C_{\mathrm{t}}=\rho C_{\mathrm{s}} \Delta s, \\
C_{\mathrm{n}}=\rho C_{\mathrm{p}} \Delta s,
\end{array}\right.
$$

where $K_{\mathrm{t}}$ and $K_{\mathrm{n}}$ are respectively the tangent and normal stiffness, $C_{\mathrm{t}}$ and $C_{\mathrm{n}}$ are respectively the tangent and normal damping, and $G$ is the shear stiffness. $R$ is the equivalent length between the source and the bottom $(5.7 \mathrm{~m})$, and $\Delta s$ is the smallest mesh size. $C_{\mathrm{s}}$ is the shear wave velocity, and $C_{\mathrm{p}}$ is the press wave velocity.

\subsection{CAM softening in the track finite difference model}

In the track finite difference model, the CAM is modeled by a solid layer. CAM softening is characterized by changing Young's modulus and the cohesion of the CAM, i.e., the parameters considered are multiplied by "softening coefficients" in the model, as follows:

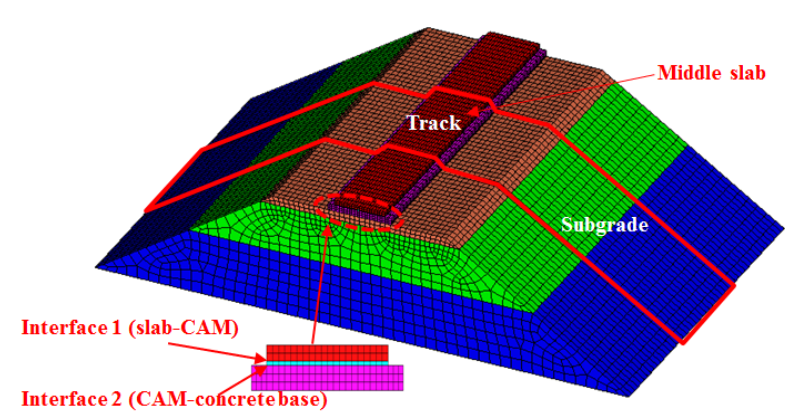

Fig. 3 3D model of a CRTS-I slab track and its subgrade

$$
\begin{cases}E_{\mathrm{CAM}}^{\prime}=E_{\mathrm{CAM}} / \lambda_{\mathrm{CAM}}, & 1 \leq \lambda_{\mathrm{CAM}}<+\infty, \\ c_{\mathrm{CAM}}^{\prime}=c_{\mathrm{CAM}} / \lambda_{\mathrm{CAM}}, & 1 \leq \lambda_{\mathrm{CAM}}<+\infty\end{cases}
$$

where $E_{\mathrm{CAM}}^{\prime}$ is the softening Young's modulus, $E_{\mathrm{CAM}}$ is the original Young's modulus, $c_{\mathrm{CAM}}^{\prime}$ is the softening cohesion, $c_{\mathrm{CAM}}$ is the original cohesion, and $\lambda_{\mathrm{CAM}}$ is the softening coefficient.

\subsection{Contact model of track}

There are two interfaces (i.e., slab-CAM, CAMconcrete base) in the track system. The interfaces of the slab-CAM and the CAM-concrete base are simulated by zero thickness elements. The constitutive relation is the Coulomb shear model. In this model, the interfaces have the properties of friction, cohesion, normal stiffness, and shear stiffness. The interface is represented as a collection of triangular elements (interface elements), each of which consists of three nodes (interface nodes). Two triangular interface elements form a quadrilateral zone face. Interface nodes are then created automatically at every interface element vertex. When another grid surface comes into contact with an interface element, the contact is detected at the interface node, and is 
characterized by normal and shear stiffnesses, and sliding properties. Each interface element distributes its area to its nodes in a weighted way. Each interface node has an associated representative area. The entire interface is thus divided into active interface nodes representing the total area of the interface as shown in Fig. 4 (Han et al., 2015). Han et al. (2015) used this contact model to study the relationship between track and subgrade surface considering water. The model in this paper is mainly used to study the CAM layer without considering water. The normal and shear forces that describe the elastic interface response are determined at the calculation time $(t+\Delta t)$ using the following relations (Han et al., 2015):

$$
\left\{\begin{array}{l}
F_{\mathrm{n}}^{t+\Delta t}=k_{\mathrm{n}} u_{\mathrm{n}} A+\sigma_{\mathrm{n}} A, \\
F_{\mathrm{si}}^{t+\Delta t}=F_{\mathrm{si}}^{t}+k_{\mathrm{s}} \Delta u_{\mathrm{si}}^{t+0.5 \Delta t} A+\sigma_{\mathrm{si}} A,
\end{array}\right.
$$

where $F_{\mathrm{n}}^{t+\Delta t}$ is the normal force at time $t+\Delta t, F_{\mathrm{si}}^{t+\Delta t}$ is the shear force at time $t+\Delta t, u_{\mathrm{n}}$ is the absolute normal penetration of the interface node into the target face, $\Delta u_{\mathrm{si}}$ is the incremental relative shear displacement, $\sigma_{\mathrm{n}}$ is the additional normal stress added due to interface stress initialization, $k_{\mathrm{n}}$ is the normal stiffness, $k_{\mathrm{s}}$ is the shear stiffness, $\sigma_{\mathrm{si}}$ is the additional shear stress due to interface stress, and $A$ is the representative area associated with the interface node initialization.

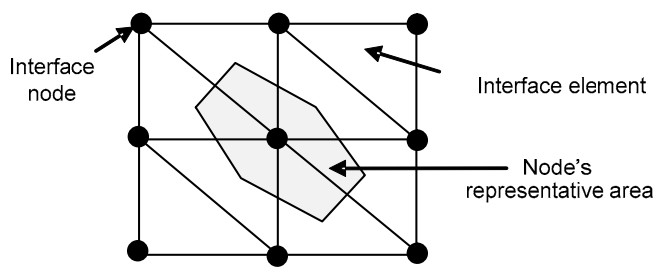

Fig. 4 Distribution of representative areas in relation to interface nodes (Han et al., 2015)

The Coulomb shear-strength criterion limits the shear force by the following relation without considering water pressure (Han et al., 2015):

$$
F_{\text {smax }}=c A+F_{\mathrm{n}} \tan \phi
$$

where $c$ is the cohesion along the interface, and $\phi$ is the friction angle of the interface surface. If the criterion is satisfied (if $\left|F_{\mathrm{s}}\right| \geq F_{\text {smax }}$ ), then sliding is assumed to occur.

\subsection{Loading on the track-subgrade finite differ- ence model}

The rail-supporting forces at fastener $i$ can be calculated using Eq. (5) by the coupling dynamic model of the vehicle and CRTS-I slab track (Section 2). The rail-supporting forces are applied to the fasteners on the slab of the $3 \mathrm{D}$ track-subgrade coupling model in Fig. 3.

$$
F_{\text {sup }, i}(t)=k_{\text {sup }} \Delta Z_{\text {sup }, i}+c_{\text {sup }} \Delta \dot{Z}_{\text {sup }}(t)
$$

where $F_{\text {sup }}$ is the discrete rail-supporting force, $k_{\text {sup }}$ and $c_{\text {sup }}$ are respectively the supporting stiffness and damping, and $\Delta Z_{\text {sup }}$ and $\Delta \dot{Z}_{\text {sup }}$ are respectively the relative displacement and the relative velocity between the rail and slab.

\section{Results and discussion}

In the analysis, the considered curved track has a radius of $7000 \mathrm{~m}$ and a super-elevation of $150 \mathrm{~mm}$. The left rail is the high rail, and the right rail is the low rail. It is assumed that different degrees of CAM softening occur when the vehicle is running on the curved track. The usual track geometry irregularity is not considered. The train speed is $300 \mathrm{~km} / \mathrm{h}$. In Sections 4.1-4.3, the CAM softening coefficients were chosen as 1 (Good: without CAM softening), 10, 100, 1000, and 10000 (Empty: the CAM has almost completely failed). Although the CAM damage condition corresponding to each CAM softening coefficient was not tested and discussed in this paper, it is very important to study the effect of the percentage of CAM softening on the dynamic behavior of the vehicletrack system. Once the influencing factors, such as track age, loading cycles, and weather cycles, are determined and shown by testing to correspond to the softening coefficient, the limit value discussed below will provide a helpful reference for the safe running of high-speed trains and track maintenance.

\subsection{Effect of CAM softening on high-speed vehicle operation safety}

Fig. 5 shows the lateral and vertical forces between the rails and the first wheelset of the vehicle when the high-speed train passes over the curved 
track with different degrees of CAM softening. The section with CAM softening is shaded in Fig. 5. When
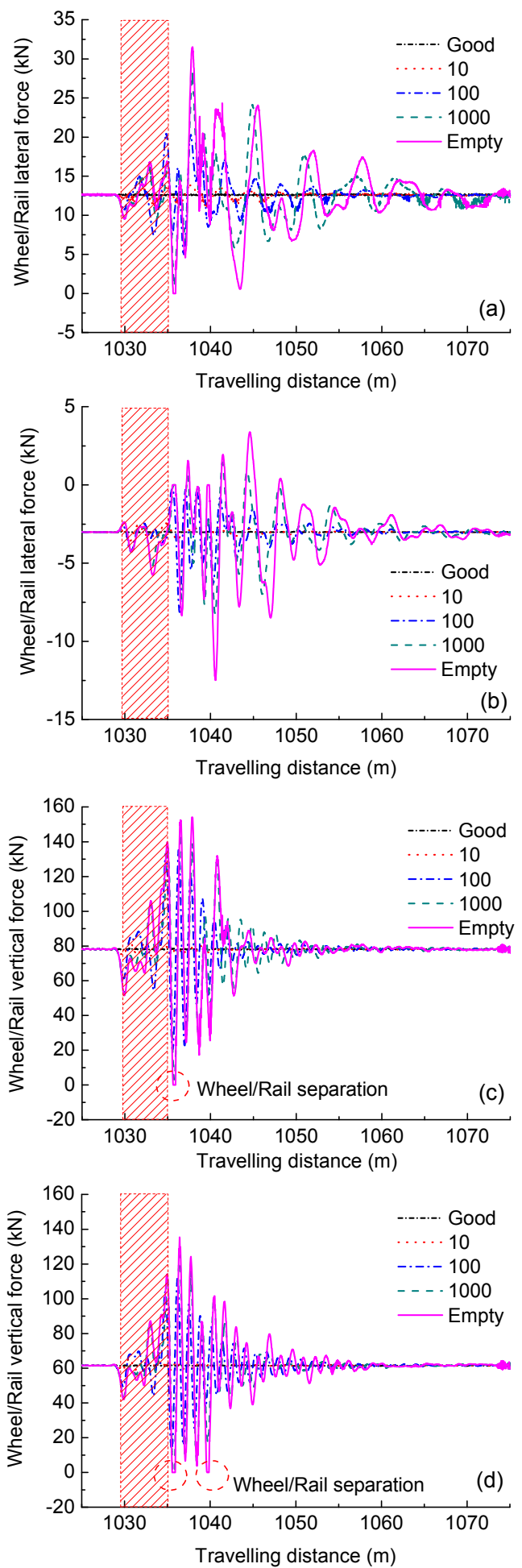

Fig. 5 Wheel/Rail forces with different degrees of CAM softening

(a) Lateral forces on high rail; (b) Lateral forces on low rail; (c) Vertical forces on high rail; (d) Vertical forces on low rail the high-speed vehicle passes through the CAM softening area, considerable impact vibrations occur between the wheels and the rails, which then gradually decay and reach a steady-state similar to that of the track without CAM softening. Fig. 5 shows clearly that the forces of the wheel/rail fluctuate dramatically in the case where CAM softening occurs on the curved track. When the vehicle passes through the track area at a CAM softening coefficient of 10000 (Empty) (when the CAM has almost completely failed), the maximum lateral and vertical forces are generated on the left wheel of the wheelset. Wheel/Rail separation is generated on both the left and right wheels when the CAM softening coefficient is larger than 1000. Due to the impact of CAM softening and the external centrifugal inertial force of the vehicle body when the train is running through the curved track, the lateral and vertical forces on the left wheel (on the high rail) are much larger than those on the right wheel of the same wheelset. Thus, the right wheel easily jumps and loses contact with the low rail, and the high-speed train risks a jumping derailment.

Fig. 6 shows the derailment coefficients $(L / V)$ with different degrees of CAM softening. The section
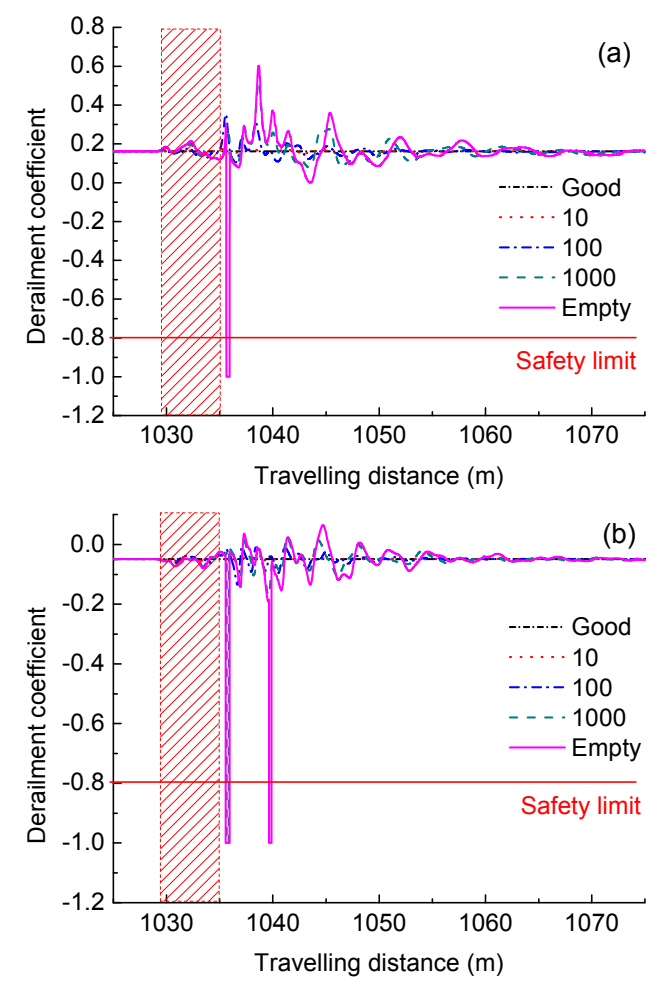

Fig. 6 Derailment coefficient $(L / V)$ with different degrees of CAM softening

(a) High rail; (b) Low rail 
with CAM softening is shaded in Fig. 6. Compared with the case without CAM softening, the absolute values of the derailment coefficient increase by about $0.02,0.19,0.36$, and 0.84 on the high rail and by about $0.01,0.10,0.96$, and 0.96 on the low rail with increasing degrees of CAM softening (Figs. 6a and $6 \mathrm{~b})$. When the softening coefficient is larger than 1000, the derailment coefficients exceed their limit value. The limit value of $L / V$ is \pm 0.8 (Zhang, 2011) according to the standard of Chinese high-speed railways.

Fig. 7 shows the wheelset loading reduction ratio $(\Delta V / V)$ with different degrees of CAM softening. Compared with the case without CAM softening, the absolute values of wheelset loading reduction increase by about $0.01,0.31,0.88$, and 0.88 with increasing degrees of CAM softening. When the softening coefficient is larger than 1000 , the wheelset loading reduction ratio exceeds its limit value. The limit value of $\Delta V / V$ is 0.6 (Zhang, 2011) according to the standard of Chinese high-speed railways.

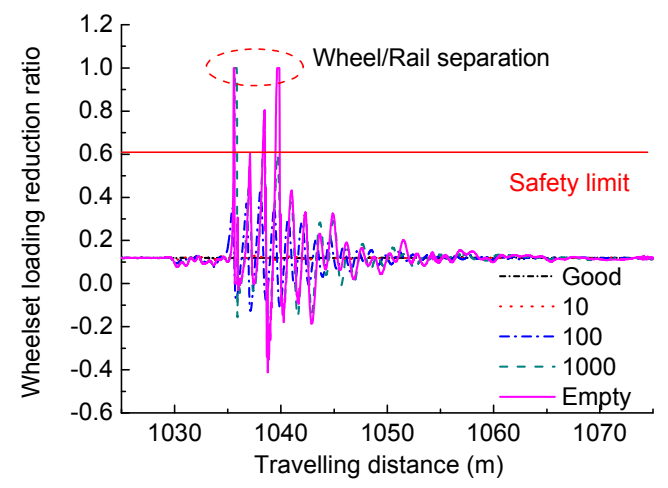

Fig. 7 Wheelset loading reduction ratio $(\Delta V / V)$ with different degrees of CAM softening

\subsection{Effect of CAM softening on track displacement}

Fig. 8 shows the rail displacement at the first wheelset in different cases of CAM softening. Compared with the case without CAM softening, the displacements of the high rail and the low rail increase as the degree of CAM softening increases. The vertical displacement of the rail is usually less than the benchmark $1.5 \mathrm{~mm}$ and should not be greater than the maximum limit of $2 \mathrm{~mm}$ (MRPRC, 2013). None of the lateral rail displacements exceed the benchmark and the maximum limit. When the softening coefficient is larger than 100 , the vertical displacement of the rail exceeds the maximum limit.
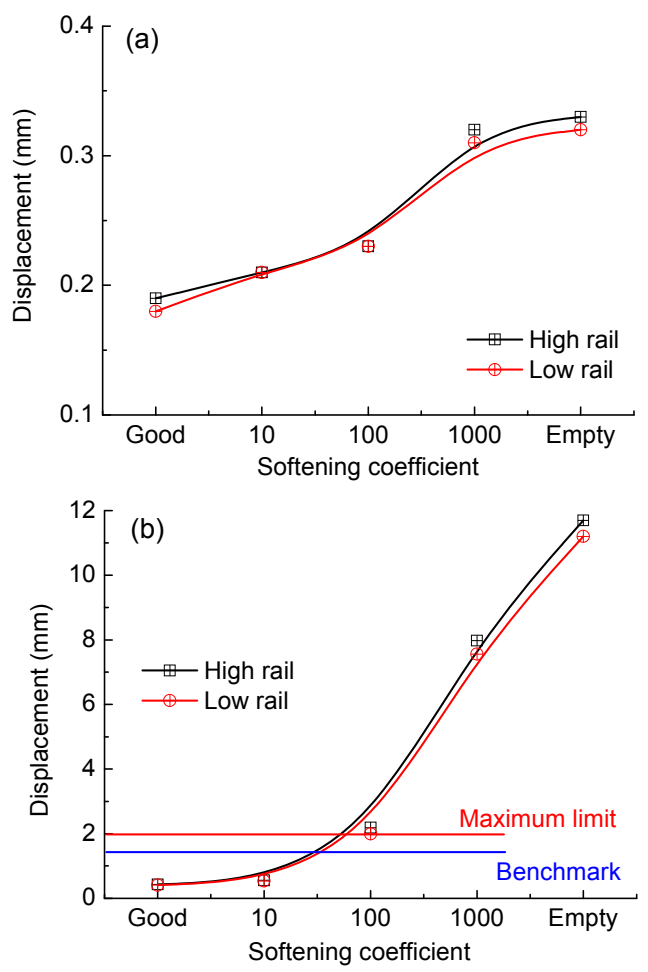

Fig. 8 Rail displacement with different degrees of CAM softening

(a) Lateral displacement; (b) Vertical displacement

Fig. 9 shows the slab displacement in different cases of CAM softening. Compared with the case without CAM softening, the displacements of the slab increase at the end and in the middle as the degree of CAM softening increases. For the slab lateral displacement, the benchmark is $0.5 \mathrm{~mm}$ and the maximum limit is $1 \mathrm{~mm}$. For the vertical slab displacement at the end, the benchmark is $0.4 \mathrm{~mm}$ and the maximum limit is $0.5 \mathrm{~mm}$. For the vertical slab displacement in the middle, the benchmark is $0.2 \mathrm{~mm}$ and the maximum limit is $0.3 \mathrm{~mm}$ (MOHURD, 2010). None of the lateral slab displacements exceed the benchmark and the maximum limit. When the softening coefficient is larger than 10 , the vertical slab displacement in the middle exceeds the corresponding benchmark. When the softening coefficient is larger than 100 , the vertical slab displacements in the middle and in the end both exceed the corresponding maximum limit.

\subsection{Effect of CAM softening on slab stress and track interface failure}

Fig. 10 shows the maximum tensile stress and shear stress of the slab in different cases of CAM 

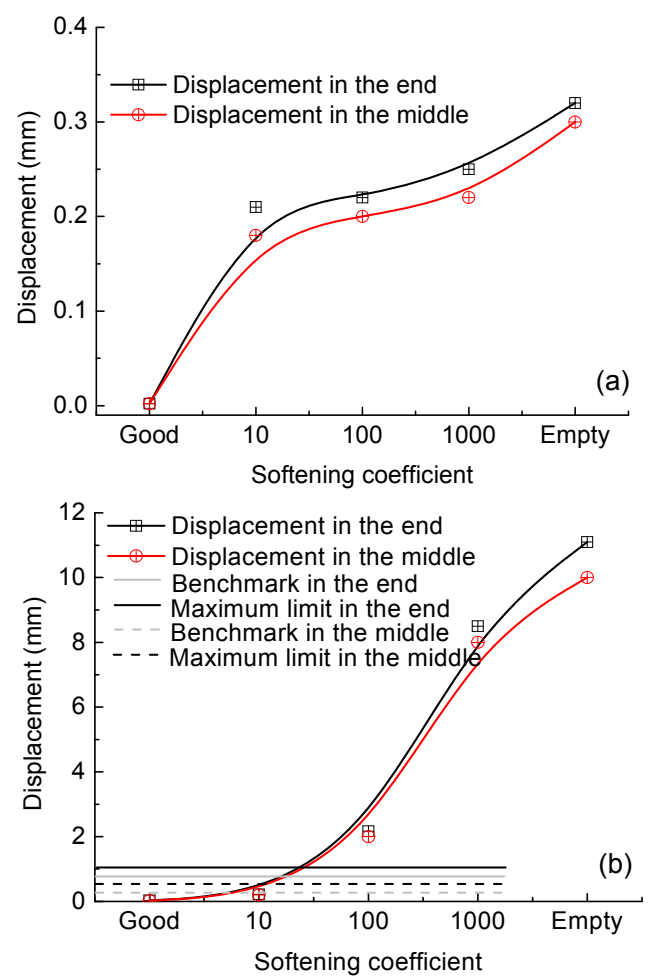

Fig. 9 Slab displacement with different degrees of CAM softening

(a) Lateral displacement; (b) Vertical displacement

softening. $\mathrm{X}, \mathrm{Y}$, and $\mathrm{Z}$ represent tensile stress in the lateral direction, longitudinal direction (travelling direction), and vertical direction, respectively. In Fig. 10a, SXX, SYY, and SZZ are tensile stresses in three directions. In Fig. 10b, SXY, SXZ, and SYZ are shear stresses in three directions. Compared with the case without CAM softening, the maximum tensile stresses and shear stresses increase as the degree of CAM softening increases. The slab material is C60 (concrete 60 ). The tensile strength is $2.85 \mathrm{MPa}$ and the shear strength is $4.1 \mathrm{MPa}$ (MOHURD, 2010). Thus, in the four cases of CAM softening, the maximum tensile stress and shear stress do not exceed their allowable strength. The compressive strength of C60 is much larger than the maximum compressive stress of the slab in the four cases of CAM softening. The compressive stresses are not given in this study.

Fig. 11a shows the interface shear failure percentage caused by CAM softening. According to Eqs. (3) and (4), when the shear force exceeds the shear-strength criterion limit, interface shear failure occurs. As the degree of CAM softening increases, the interface shear failure percentage increases slowly when the softening coefficient is larger than 1000 or
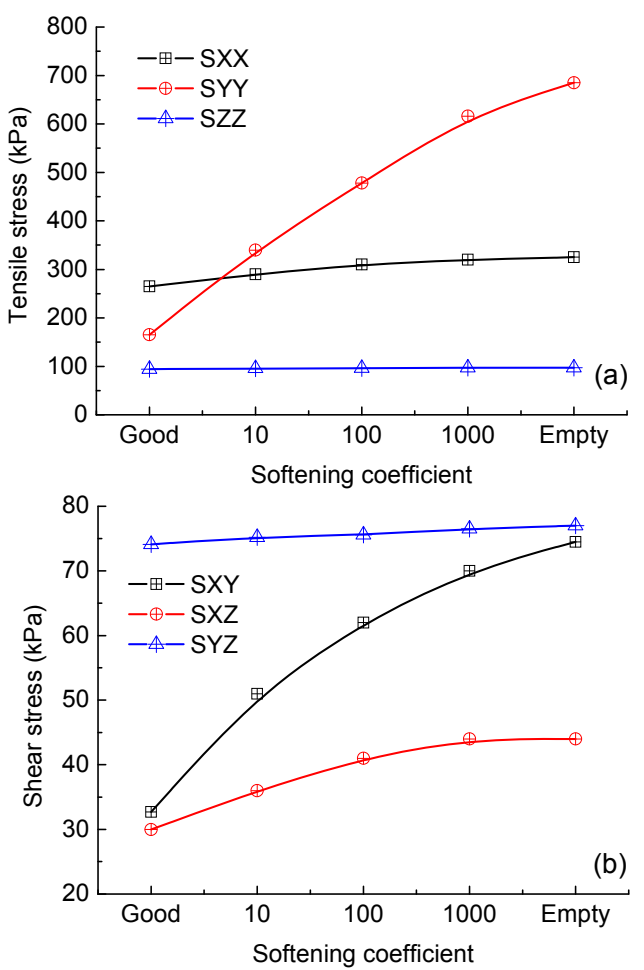

Fig. 10 Slab stress with different degrees of CAM softening: (a) tensile stress; (b) shear stress

(a)
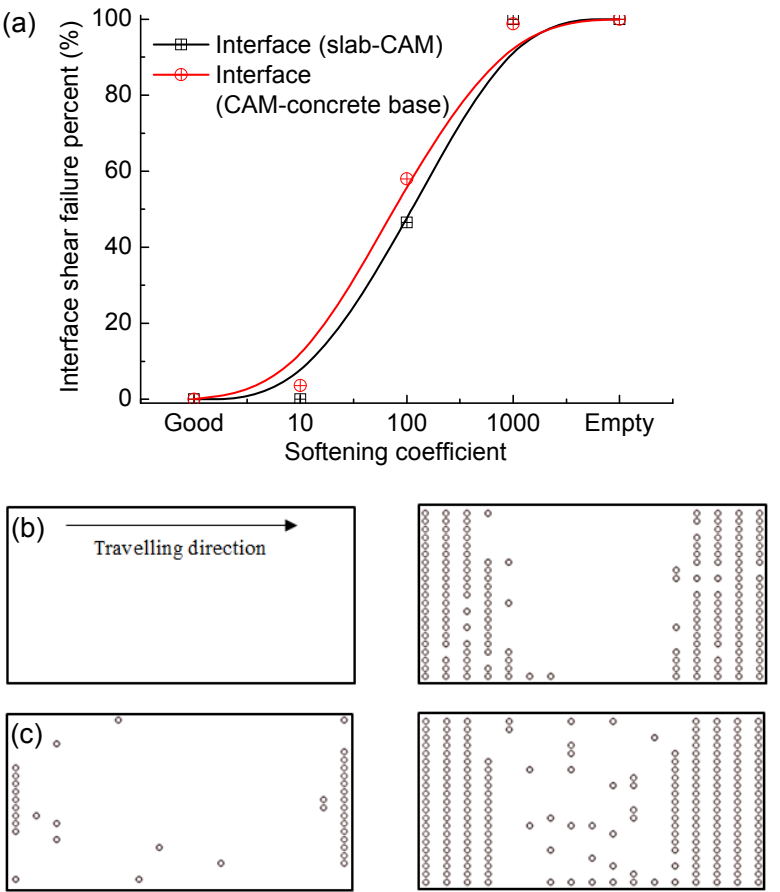

Fig. 11 Interface shear failure

(a) Interface shear failure percent vs CAM softening coefficient; (b) Interface shear failure distribution (slab-CAM) (left: 10; right: 100); (c) Interface shear failure distribution (CAMconcrete base) (left: 10; right: 100) 
smaller than 10, and increases quickly when the softening coefficient is between 10 and 1000 . The relationship between interface shear failure percentage and the softening coefficient can be fitted using the GaussAmp Formula (Amplitude version of Gaussian peak function). The whole fitting curve is similar to an S-shaped curve. Compared with the interface between the slab and CAM, the interface between CAM and the concrete base is more vulnerable to shear failure (Fig. 11a). Figs. 11b and 11c show the interface shear failure distributions corresponding to softening coefficients 10 and 100 . When the softening coefficient is 10 , the interface between the slab and the CAM does not show shear failure, and the interface between the CAM and the concrete base shows only a small partial shear failure. However, when the softening coefficient is 100 , the shear failure percentages of both the interfaces between the slab and the CAM and between the CAM and the concrete base, reach about $45 \%-60 \%$. The failure percentage of the CAM-concrete base interface is higher than that of the slab-CAM interface because the cohesion of the interface between the CAM and the concrete base is smaller. When the softening coefficients change from 10 to empty, the gap between the CAM-concrete base and the slab-CAM lines decreases. As this progresses, as discussed above, the interface between the CAM and the concrete base fails first. With the failure increasing significantly, even reaching complete failure, lateral movement of the slab may easily occur. This lateral movement of the slab will then speed up the relative motion between the slab and the CAM, increasing the risk of interface failure. Finally, the interface between the CAM-concrete base and the interface between the slab-CAM fails completely. Figs. $11 \mathrm{~b}$ and $11 \mathrm{c}$ also show that the interface shear failure develops from the end to the middle. This is because the relative shear displacement at the end is larger than that in the middle, which leads to a larger shear force at the end. The shear force at the end then more easily exceeds the shear-strength limit.

\section{Conclusions}

In this paper, a 3D coupling dynamic model of a vehicle and a CRTS-I slab track is developed. Using the proposed model, the wheel-rail contact forces, derailment coefficient, wheelset loading reduction ratio, and the track displacements are calculated to study the influence of CAM softening on the dynamic characteristics of the vehicle-track system. A track-subgrade finite difference model is developed to study the effect of CAM softening on track damage. The following conclusions can be drawn:

1. Wheel-rail contact forces fluctuate dramatically when a high-speed train runs over the curved track with CAM softening. When the CAM softening coefficient is larger than 1000 , wheel/rail separation occurs, and the derailment coefficient and wheelset loading reduction ratio both exceed their safety limits.

2. As CAM softening increases, slab displacement more easily exceeds its geometric limit than rail displacement. When the CAM softening coefficient is larger than 10 , slab vertical displacement in the middle exceeds the corresponding benchmark. When the softening coefficient is larger than 100 , the vertical displacements of both the rail and slab exceed their corresponding maximum limits.

3. CAM softening cannot lead to slab damage based on a simple strength analysis. When the CAM softening coefficient reaches 10 , a small partial slip occurs between the CAM and the concrete base. When the CAM softening coefficient is larger than 100 , at both the slab-CAM interface and the CAM-concrete base interface, serious damage occurs due to slippage.

According to these conclusions, when the CAM softening coefficients reach 10-100, track interface shear failure develops. The CAM softening coefficient should not be less than 1000 , otherwise a high-speed running vehicle may risk derailment.

In future work, we propose to conduct a series of tests to obtain the relationship between the softening coefficients of CAM and loading cycles, temperature cycles, and weather conditions.

\section{References}

Chen, G., Zhai, W.M., 2004. A new wheel/rail spatially dynamic coupling model and its verification. Vehicle System Dynamics, 41(4):301-322. [doi:10.1080/00423110412 331315178]

Chen, R.P., Chen, J.M., Wang, H.L., 2014. Recent research on the track-subgrade of high-speed railways. Journal of Zhejiang University-SCIENCE A (Applied Physics \& Engineering), 15(12):1034-1038. [doi:10.1631/jzus. A1400342]

Han, J., Zhao, G.T., Xiao, X.B., et al., 2015. Contact behaviour between slab track and its subgrade under high-speed train loading and water-soil interaction. Electronic Journal of Geotechnical Engineering, 20(2):709-722.

Jin, X.S., 2014. Key problems faced in high-speed train 
operation. Journal of Zhejiang University-SCIENCE A (Applied Physics \& Engineering), 15(12):936-945. [doi:10.1631/jzus.A1400338]

Jin, X.S., Wen, Z.F., 2008. Effect of discrete track support by sleepers on rail corrugation at a curved track. Journal of Sound and Vibration, 315(1-2):279-300. [doi:10.1016/j. jsv.2008.01.057]

Lin, H.S., 2009. Research on the Static and Dynamic Property of Ballastless Track based on Fracture and Damage Mechanics. PhD Thesis, Southwest Jiaotong University, Chengdu, China (in Chinese).

Ling, L., Xiao, X.B., Xiong, J.Y., et al., 2014. A 3D model for coupling dynamics analysis of high-speed train/track system. Journal of Zhejiang University-SCIENCE A (Applied Physics \& Engineering), 15(12):964-983. [doi:10.1631/jzus.A1400192]

Liu, J.B, Gu, Y., Du, Y.X., 2006. Consistent viscous-spring artificial boundaries and viscous-spring boundary elements. Chinese Journal of Geotechnical Engineering, 28(9):1070-1075 (in Chinese).

Liu, Y., 2013. Study on Characteristics and Influences of CRTS II Slab Track Early Temperature Field. PhD Thesis, Southwest Jiaotong University, Chengdu, China (in Chinese).

MOHURD (Ministry of Housing and Urban-Rural Development of the People's Republic of China), 2010. Code for Design of Concrete Structures, GB 50010-2010. China Architecture and Building Press, Beijing, China (in Chinese).

MRPRC (Ministry of Railways of the People's Republic of China), 2013. Technical Regulations for Dynamic Acceptance for High-speed Railways Construction, TB 10716-2013. Railway Publishing House, Beijing, China (in Chinese).

Shen, Z.Y., Hedrick, J.K., Elkins, J.A., 1983. A comparison of alternative creep-force models for rail vehicle dynamic analysis. Vehicle System Dynamics, 12(1-3):79-83. [doi:10.1080/00423118308968725]

Wang, P., Xu, H., Chen, R., 2014. Effect of cement asphalt mortar debonding on dynamic properties of CRTS II slab ballastless track. Advances in Materials Science and Engineering, 2014:193128. [doi:10.1155/2014/193128]

Xiang, J., He, D., Zeng, Q.Y., 2009. Effect of cement asphalt mortar disease on dynamic performance of slab track. Journal of Central South University, 40(3):791-796 (in Chinese).

Xiao, X.B., Jin, X.S., Wen, Z.F., 2007. Effect of disabled fastening systems and ballast on vehicle derailment. Journal of Vibration and Acoustics, 129(2):217-229. [doi:10.1115/1.2424978]

Xiao, X.B., Jin, X.S., Deng, Y.Q., et al., 2008. Effect of curved track support failure on vehicle derailment. Vehicle System Dynamics, 46(11):1029-1059. [doi:10.1080/ 00423110701689602]

Xiao, X.B., Jin, X.S., Wen, Z.F., et al., 2011. Effect of tangent track buckle on vehicle derailment. Multibody System Dynamics, 25(1):1-41. [doi:10.1007/s11044-010-9210-2]

Xiao, X.B., Ling, L., Xiong, J.Y., et al., 2014. Study on the safety of operating high-speed railway vehicles subjected to crosswinds. Journal of Zhejiang University-SCIENCE
A (Applied Physics \& Engineering), 15(9):694-710. [doi:10.1631/jzus.A1400062]

Zhai, W.M., 1996. Two simple fast integration methods for large-scale dynamic problems in engineering. International Journal for Numerical Methods in Engineering, 39(24):4199-4214.

Zhang, W.H., 2011. Overall Technique of EMU and Bogies. Chinese Railway Press, Beijing, China (in Chinese).

Zhong, S.Q., Xiong, J.Y., Xiao, X.B., et al., 2014. Effect of the first two wheelset bending modes on wheel-rail contact behavior. Journal of Zhejiang University-SCIENCE A (Applied Physics \& Engineering), 15(12):984-1001. [doi:10.1631/jzus.A1400199]

Zhou, L., Shen, Z.Y., 2013. Dynamic analysis of a high-speed train operating on a curved track with failed fasteners. Journal of Zhejiang University-SCIENCE A (Applied Physics \& Engineering), 14(6):447-458. [doi:10.1631/ jzus.A1200321]

Zhu, S.Y., Cai, C.B., 2014. Interface damage and its effect on vibrations of slab track under temperature and vehicle dynamic loads. International Journal of Non-Linear Mechanics, 58:222-232. [doi:10.1016/j.ijnonlinmec. 2013.10.004]

Zhu, S.Y., Fu, Q., Cai, C.B., et al., 2014. Damage evolution and dynamic response of cement asphalt mortar layer of slab track under vehicle dynamic load. Science China Technological Sciences, 57(10):1883-1894. [doi:10.1007/ s11431-014-5636-8]

\section{中文概要}

\section{题 目: 沥青混凝土砂浆层软化对车辆行驶安全性和轨道} 动力特性的影响

目 的: 对于高速铁路, 老化和雨水冲刷浸泡将导致无砟 轨道水泥沥青砂浆 (CA 砂浆) 软化现象。CA 砂 浆的软化严重影响轨道动力特性, 甚至危害高速 列车行车安全。通过仿真计算, 系统地调查 CA 砂浆软化对高速列车行车安全以及轨道动力特 性的影响。

创新点: 系统地对比 $\mathrm{CA}$ 砂浆软化对车辆-轨道动力学特性 和轨道层间破坏的影响, 为工程实际提供参考。

方 法: 1. 通过建立三维车辆-轨道耦合动力学模型以及 $\mathrm{CA}$ 砂浆软化模型, 分析 CA 砂浆软化对行车安全 以及轨道动力特性的影响 (图 2); 2. 通过轨道路基非线性有限差分耦合模型, 分析 CA 砂浆软 化对轨道层间破坏的影响（图 3)。

结 论: 通过车辆运行安全性分析、轨道位移限值分析以 及轨道层间失效分析, 得出以下结论: 1. CA 砂 浆软化系数达到 10 100 时, 轨道层间剪切失效 开始快速发展; 2. CA 砂浆软化系数不能超过 1000 , 当 CA 砂浆软化系数超过该值时, 高速列 车将面临脱轨危险。

关键词: 高速铁路; $\mathrm{CA}$ 砂浆软化; 车辆-轨道耦合动力学; 运行安全性; 轨道破坏 